\title{
Reproducción de hembras de la langosta Panulirus inflatus (Bouvier, 1895) en el litoral del Pacífico de México
}

\author{
Reproduction of females lobster Panulirus inflatus (Bouvier, 1895) \\ from the Pacific coast of Mexico
}

\section{Ma. Guadalupe Torres Zepeda1, Juan Carlos Zepeda Castillo', Juan Antonio Meza García ${ }^{1}$, Adolfo Solís Hernández ${ }^{1}$ Marcial Villalejo Fuerte ${ }^{2}$}

\author{
${ }^{1}$ Universidad Autónoma de Guerrero, Unidad académica de Ecología Marina, Acapulco Guerrero, México, \\ Avenida Gran Vía Tropical No 20, Fraccionamiento Las Playas. \\ ${ }^{2}$ CICIMAR-IPN, La Paz Baja California Sur, México. Avenida Instituto Politécnico Nacional s/n, \\ Colonia Playa Palo de Santa Rita \\ mariagua66@hotmail.com
}

\begin{abstract}
The spiny lobster $P$. inflatus is a fishing resource of regional economic importance in Guerrero, Mexican Tropical Pacific coast where this lobster has not been studied enough. This analysis of the captures in Guerrero's coasts will contribute to a better managing of this fishery. We studied the size distribution of the capture, the percentages of reproductive females and the size class by climatic season, as well as the size of sexual maturity in 2569 lobsters collected from June 2004 to July 2005 in three zones in the Guerrero's coastal region: Copala, Zihuatanejo and Acapulco. The size composition and the size contributing with major number of lobsters to this commercial catch differed among the study sites. The wider range and the bigger sizes were obtained in Acapulco followed by Zihuatanejo and, finally, by Copala. There were no significant differences for the percentages of reproductive females among between stations in the three places $\left(\chi^{2}, P>0.05\right)$ and the reproductive females (with spermatophores and/or ovigerous mass) grouped by size class, in general presented maximums in summer and autumn exempting the sizes under $50 \mathrm{~mm}$ LC and the ones over $80 \mathrm{~mm}$ LC. Size at first sexual maturity in $P$. inflatus was $57.4 \mathrm{~mm} \mathrm{LC}$ at Copala, $63.6 \mathrm{~mm}$ LC at Zihuatanejo and $68.6 \mathrm{~mm} \mathrm{LC}$ at Acapulco. The results indicate the necessity to manage the resource taking into account the differences among capture areas.
\end{abstract}

Key words: Spawning season, size at sexual maturity, size distribution

\section{Introducción}

La producción de langostas en México está compuesta por cinco especies que se encuentran en aguas tropicales y subtropicales, cuatro de ellas en las costas del Pacífico (Panulirus interruptus, $P$. inflatus, $P$. gracilis y $P$. penicillatus) y una en el caribe mexicano ( $P$. argus) (DíazArredondo \& Guzmán-del-Próo 1995). P. inflatus es endémica de las costas del Pacífico mexicano y se distribuye desde Isla Margarita en Baja California Sur
Resumen.- La langosta espinosa $P$. inflatus es un recurso pesquero de importancia económica regional en el litoral del Pacífico mexicano que ha sido poco estudiada. Este análisis del monitoreo de capturas en las costas del estado de Guerrero aporta conocimientos que contribuirán a un mejor manejo de esta pesquería. Se estudió la composición de tallas, el porcentaje de hembras reproductivas por estación climática y por clase de talla, así como la talla de madurez sexual en 2569 langostas recolectadas de junio 2004 a julio 2005, en tres zonas de captura ubicadas en las regiones costeras de Guerrero: Copala, Zihuatanejo y Acapulco. La composición de tallas y la talla que aporta el mayor número de organismos en estas capturas difieren entre los sitios de estudio, obteniéndose el rango más amplio y la talla más grande en Acapulco, seguido de Zihuatanejo y, por último, en Copala. No hubo diferencias significativas en los porcentajes de hembras reproductivas entre estaciones en los tres sitios $\left(\chi^{2}, P>0,05\right)$; las hembras reproductivas (con espermatóforo y/o masa ovígera), agrupadas por clase de tallas, presentaron, en general, máximos en verano y otoño, exceptuando las tallas menores a $50 \mathrm{~mm}$ de LC y las mayores a $80 \mathrm{~mm}$ de LC. La talla de primera madurez sexual de $P$. inflatus fue de 57,4 mm de LC en Copala, de 63,6 mm de LC en Zihuatanejo y de 68,6 mm de LC en Acapulco. Los resultados indican la necesidad de manejar el recurso tomando en cuenta las diferencias entre zonas de captura.

Palabras clave: Época reproductiva, talla de madurez, composición de tallas

incluyendo el Golfo de California, hasta las costas del Golfo de Tehuantepec (Holthuis \& Villalobos 1962). La pesca ribereña de langosta en el litoral de Guerrero está compuesta por $P$. inflatus y $P$. gracilis, especies que coexisten en la mayor parte de las costas del Pacífico mexicano (Briones-Fourzán \& Lozano-Álvarez 1992).

$P$. inflatus y $P$. gracilis son localmente importantes como recurso pesquero, llegando a representar del 9,7 al 17,3\% de la producción total de langosta espinosa en 
México, de 1981 a 1988 (Briones-Fourzán \& LozanoÁlvarez 1992).

Dada la importancia de este recurso en las pesquerías del Pacífico mexicano y que los estudios realizados en las costas de Guerrero (Briones et al. 1981, BrionesFourzán \& Lozano-Álvarez 1992, Briones-Fourzán \& Lozano-Álvarez 2003, Gracia 1985, Weinborn 1977, Lozano-Álvarez \& Aramori-Serrano 1997, Lozano et al. 1982 y Briones et al. 1981) habían sido realizados solo en Zihuatanejo y datan de mas de 20 años, se realizó este estudio con el objetivo de analizar la época de desove, desove de diferentes grupos de talla y estimar la talla mínima de madurez sexual de la langosta $P$. inflatus a partir de muestreos realizados en las capturas comerciales, de junio de 2004 a julio de 2005, en tres zonas del litoral del estado de Guerrero, México. Adicionalmente se comparan y discuten los resultados obtenidos en 19791980 y se revisan las disposiciones legales relacionadas con la captura de estos organismos con el fin de aportar información para una mejor administración del recurso.

\section{Materiales y métodos}

\section{Área de estudio}

Las zonas de pesca de Panulirus inflatus (Bouvier, 1895) que se consideraron en este estudio en la costa del estado de Guerrero, México, se localizan en tres regiones: la región Costa Chica donde la zona de captura se realiza en el municipio de Copala desde los $16^{\circ} 30^{\prime} 36^{\prime \prime} \mathrm{N}$ y $98^{\circ}$ $55^{\prime} 35^{\prime \prime W}$ hasta los $16^{\circ} 25^{\prime} 41^{\prime \prime} \mathrm{N}$ y $98^{\circ} 40^{\prime} 30^{\prime \prime W}$. La región Costa Grande, que abarca desde la localidad de pesca conocida como El Riscal (17\%35'11'”N $\left.101^{\circ} 26^{\prime} 34^{\prime \prime} \mathrm{W}\right)$ a la Salada o Saladita $\left(17^{\circ} 50^{\prime} 21^{\prime \prime} \mathrm{N}\right.$ $101^{\circ} 46^{\prime} 04^{\prime \prime}$ 'W), incluyendo la bahía de Zihuatanejo e isla Ixtapa, en el municipio Teniente José Azueta y la tercera región en el municipio de Acapulco, donde las capturas se realizan desde la Colonia Azteca (1653'20”'N - 9950'30”'W) hasta los Morros Rojos (1646'45” $\mathrm{N}$ - 9950'30”'W), comprendiendo las bahías de Acapulco y Puerto Marquez, así como la isla La Roqueta (Fig. 1).

\section{Muestreo}

A partir de junio de 2004 y hasta mayo de 2005, se registraron mensualmente los datos de todos los organismos obtenidos por viaje en la captura comercial. Los organismos fueron extraídos por buceo libre, autónomo o semiautónomo utilizando un gancho de acero.

\section{Distribución de tallas de las capturas}

De cada ejemplar se registró el sexo y se midió la longitud del cefalotórax (LC) utilizando un vernier de 12 cm, con precisión de 0,01 cm. La distribución de tallas de las capturas se obtuvo en intervalos de $5 \mathrm{~mm}$ de LC, para ambos sexos (langostas hembras, hembras grávidas y machos) capturados durante el ciclo de estudio, por zona de captura.

\section{Estimación de la estación climática de mayor actividad reproductiva}

La estimación de la estación de mayor actividad reproductiva se analizó mediante una prueba estadística de $\div^{2} \quad(a ́=0,05)$ (Wayne, 2005) aplicada al porcentaje de hembras grávidas por estación climática y por área de estudio. Adicionalmente se estimó el porcentaje por clase de talla agrupando a las hembras grávidas en intervalos de tallas de $10 \mathrm{~mm}$ de LC, por estación y área de pesca.

\section{Talla mínima de madurez sexual de las hembras}

Considerada como la talla en la cual el $50 \%$ de las hembras presenta espermatóforo y/o tienen huevos en incubación. Se determinó a través de un ajuste a un modelo logístico (Somerton 1980).

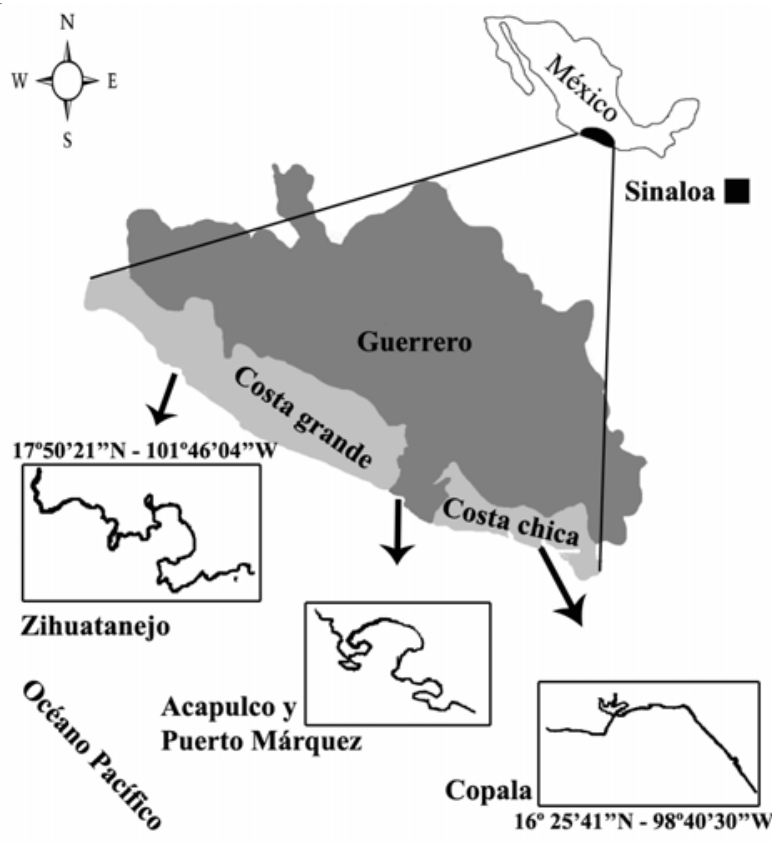

Figura 1

Zonas de captura de la langosta espinosa $P$. inflatus en la costa de Guerrero, Pacífico mexicano, estudiadas durante el periodo junio 2004-mayo 2005

Capture areas of the spiny lobster $P$. inflatus in the coast of Guerrero, Mexican Pacific, studied during the June 2004May 2005 period 


\section{Resultados}

Se capturaron en total 2569 langostas de $P$. inflatus en las tres áreas de estudio: 823 en Copala, 790 en Zihuatanejo y 956 en Acapulco. El número de hembras, machos y la proporción de sexo por área y arte de pesca se muestran en la Tabla 1. Las temperaturas registradas en el mar en el litoral de Guerrero en general muestran un incremento hasta $\operatorname{los} 30,5^{\circ} \mathrm{C}$ en verano, disminuye en uno o dos grado en otoño e invierno y en primavera se inicia el descenso de temperaturas hasta $21^{\circ} \mathrm{C}$ en los meses de abril y mayo.

\section{Composición de tallas de las capturas}

El rango de tallas en las capturas por área de estudio así como la talla de mayor incidencia de langostas y de mayor número de hembras se indican en la Fig. 2.

Respecto a la captura de hembras por área, en Copala y Zihuatanejo el porcentaje de hembras con huevos representó el 39,9\% y 39,6\% respectivamente, y en Acapulco fue de $47,6 \%$.

\section{Estación de mayor actividad reproductiva}

Los porcentajes de hembras grávidas por estación en las áreas de estudio se muestran en la Fig. 3 y la Tabla 2. La diferencia en el porcentaje de hembras grávidas entre estaciones no fue significativa $(P>0,05)$.

La composición estacional de hembras reproductivas por clase de talla mostró que en Acapulco las hembras ovígeras de tallas menores a $50 \mathrm{~mm}$ de LC tuvieron un mayor porcentaje en invierno, mientras que en Copala, el porcentaje fue similar en invierno y otoño. Las hembras entre 50 y 60 mm de LC presentaron un máximo en otoño en las tres localidades. De 60 a $70 \mathrm{~mm}$ de LC se observaron todo el año en Copala, mientras que en Zihuatanejo y Acapulco tuvieron porcentajes mayores en otoño. De 70 a $80 \mathrm{~mm}$ de LC, la mayor abundancia se

\section{Tabla 1}

Número total de hembras y machos capturados por área de estudio y proporción de sexos de $P$. inflatus, en la costa de Guerrero, Pacífico mexicano, desde junio de 2004 a mayo de 2005

Total number of females and males captured by study area and proportion of sexes of the spiny lobster $P$. inflatus, in the Guerrero coast, Mexican Pacific, from June 2004 to May 2005

\begin{tabular}{cccccr}
\hline Area & Arte de captura & Hembras & Machos & Total & H:M \\
\hline Copala & Compresor & 407 & 416 & 823 & $1: 1$ \\
Zihuatanejo & Compresor & 331 & 459 & 790 & $1,4: 1$ \\
Acapulco & Scuba y libre & 498 & 458 & 956 & $0,9: 1$ \\
\hline
\end{tabular}
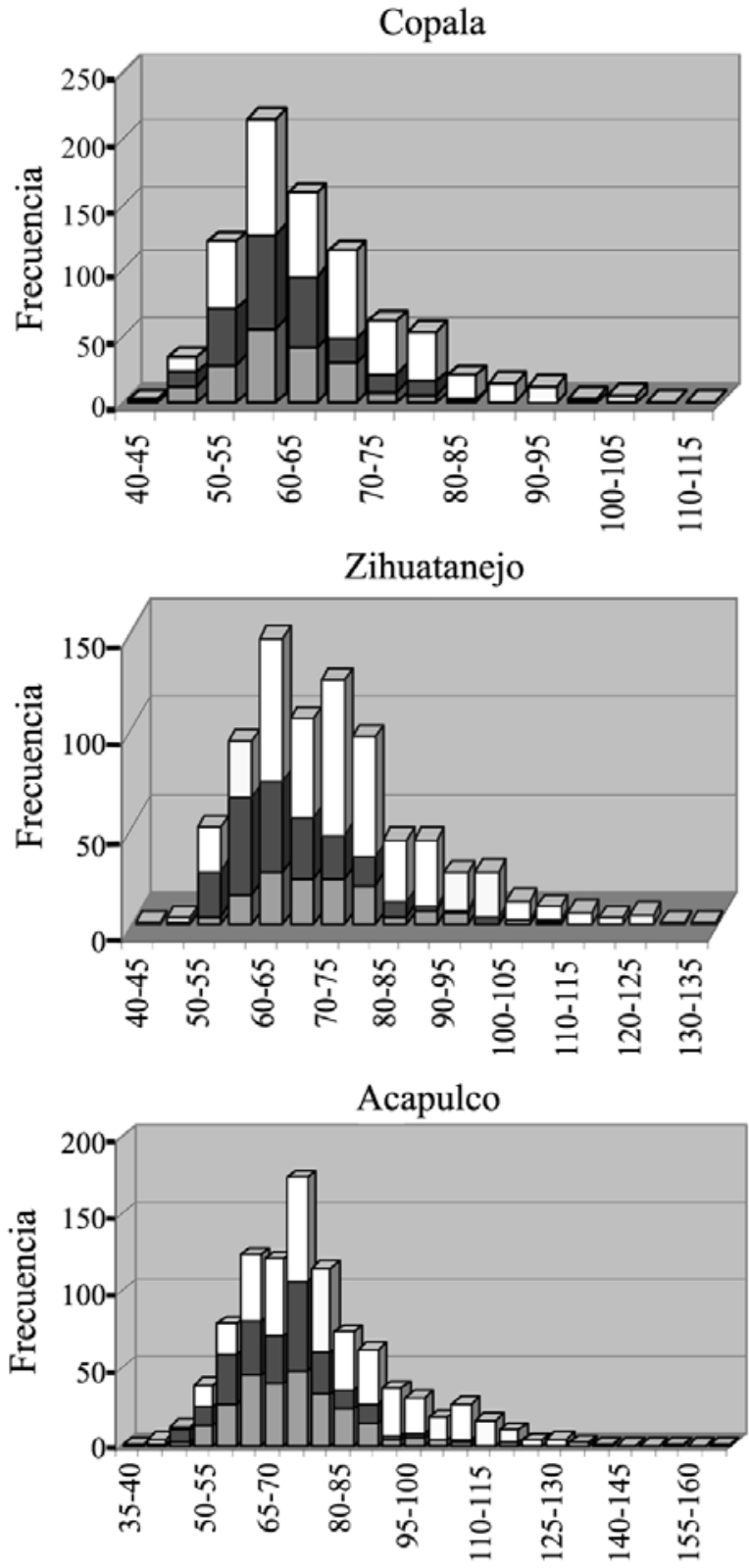

Clase de talla en mm de LC

"Hem Ovígeras $\approx$ Hem 1 y 6 a Machos

Figura 2

Distribución de tallas (en frecuencias de $5 \mathrm{~mm}$ longitud cefalotoráxica LC) de la langosta espinosa $P$. inflatus en las zonas de estudio, en la costa de Guerrero, Pacífico mexicano, durante 2004-2005

Size distribution (in frequencies of $5 \mathrm{~mm}$ cephalotoraxic length LC) of spiny lobster $P$. inflatus in the study areas of the Guerrero coast, Mexican Pacific, during 2004-2005 


\section{Tabla 2}

Porcentaje estacional de hembras con huevos en las tres áreas de la pesquería de $\boldsymbol{P}$. inflatus en las costas de Guerrero, del Pacífico mexicano durante el estudio de junio 2004-mayo 2005

Seasonal percentage of females with eggs in the three study areas of the spiny lobster $P$. inflatus fishery in the Guerrero coast, Mexican Pacific, from June 2004 to May 2005

\begin{tabular}{ccccccc}
\hline Estación & Copala & $\begin{array}{c}\mathrm{X}^{2} \\
P=0,05\end{array}$ & Zihuatanejo & $\begin{array}{c}\mathrm{X}^{2} \\
P=0,05\end{array}$ & Acapulco & $\begin{array}{c}\mathrm{X}^{2} \\
P=0,05\end{array}$ \\
\hline Verano & 46,58 & 0,1282 & 50,5 & 1,1195 & 50,99 & 0,0401 \\
Otoño & 48,09 & 0,3424 & 55,4 & $\mathbf{3 , 2 4 3 0}$ & 55,26 & 0,516 \\
Invierno & 42,40 & 0,0733 & 34,7 & 0,8606 & 44,86 & 1,0957 \\
Primavera & 39,74 & 0,4500 & 30,8 & $\mathbf{3 , 7 1 7 8}$ & 58,68 & 0,7425 \\
\hline
\end{tabular}

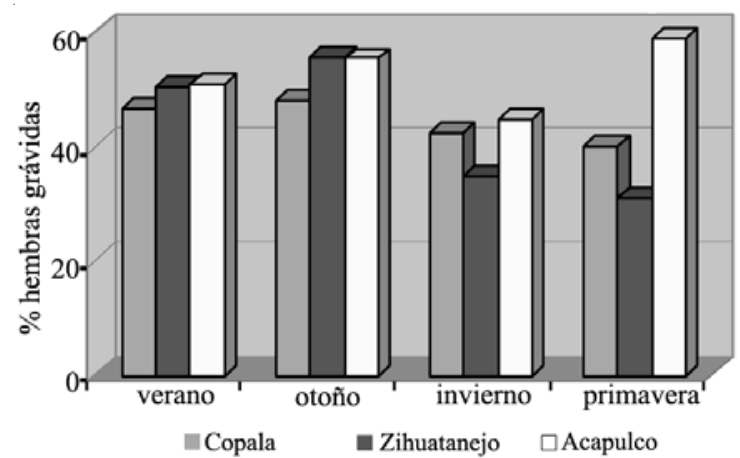

Figura 3

Porcentaje de hembras grávidas de langosta espinosa $P$. inflatus en cada estación en las tres zonas de captura pesquera en la costa de Guerrero, Pacífico mexicano, durante el periodo 2004-2005

Percentage of berried females of the spiny lobster $P$. inflatus in each station at the three areas of fishery capture in the Guerrero coast, Mexican Pacific, during the period 2004-2005

registró en verano en Copala y se presentaron durante todo el año en Zihuatanejo y Acapulco. Hembras mayores de $80 \mathrm{~mm}$, presentaron máximos en otoño en Copala y Zihuatanejo y en primavera en Acapulco (Fig. 4).

\section{Talla mínima de madurez sexual de las hembras}

Los valores obtenidos por zona de captura fueron los siguientes: en Copala 57,4 mm de LC, en Zihuatanejo 63,58 mm de LC y en Acapulco 68,59 mm de LC (Fig. 5).

\section{Discusión}

\section{Actividad reproductiva y periodo de veda}

Es relativamente fácil establecer las temporadas de veda en pesquerías de especies de langostas del género
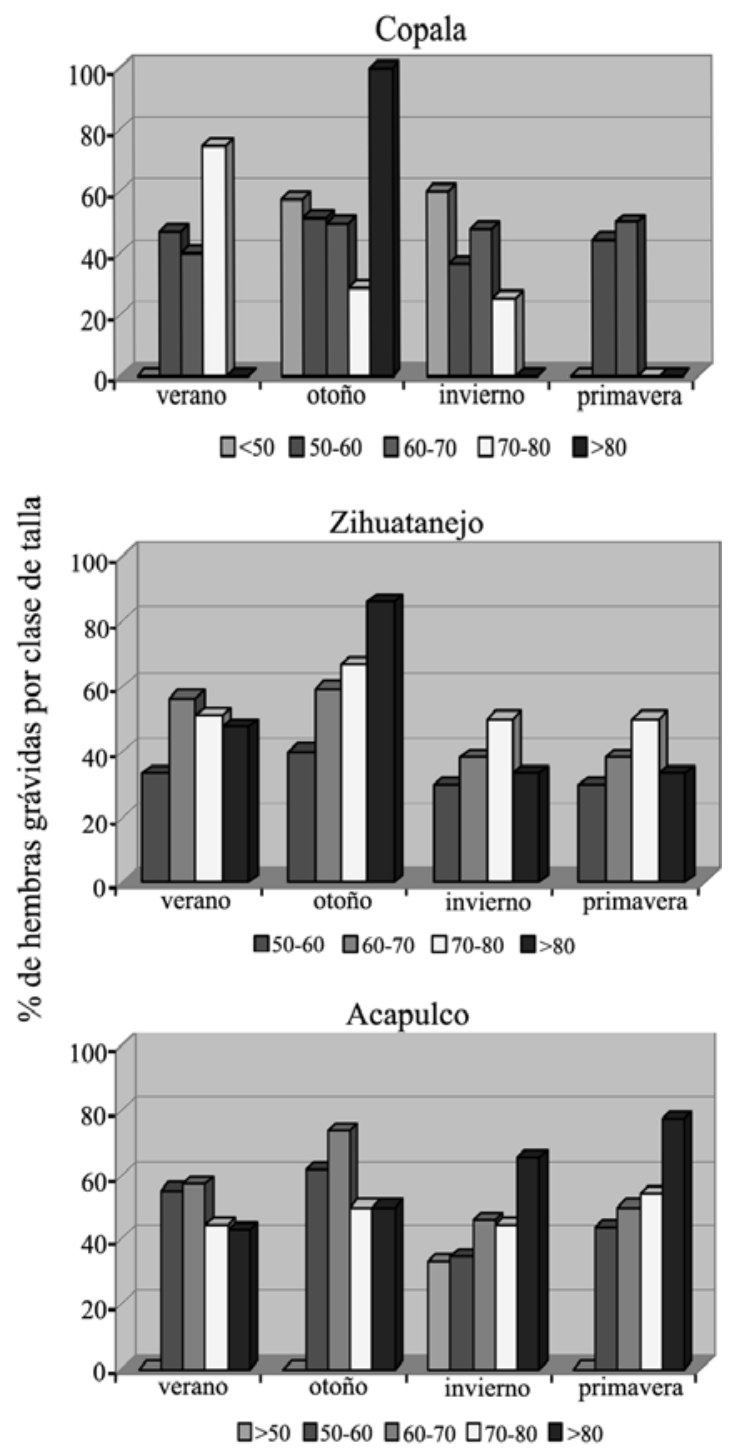

Figura 4

Incidencia estacional (en \%) de hembras reproductivas por clase de talla (en mm LC) para la langosta espinosa $P$.

inflatus en Copala, Zihuatanejo y Acapulco, costa de Guerrero, Pacífico mexicano

Seasonal incidence (in \%) of berried females by size class (in mm LC) for of the spiny lobster P. inflatus in Copala, Zihuatanejo and Acapulco, Guerrero coast, Mexican Pacific

Panulirus en climas templados y subtropicales en donde presentan estaciones reproductivas bien definidas o la aplicación de la temporada de veda por zonas cuando presentan variaciones latitudinales en el inicio de la actividad reproductiva como $P$. interruptus en Baja California (Pineda et al. 1981). 


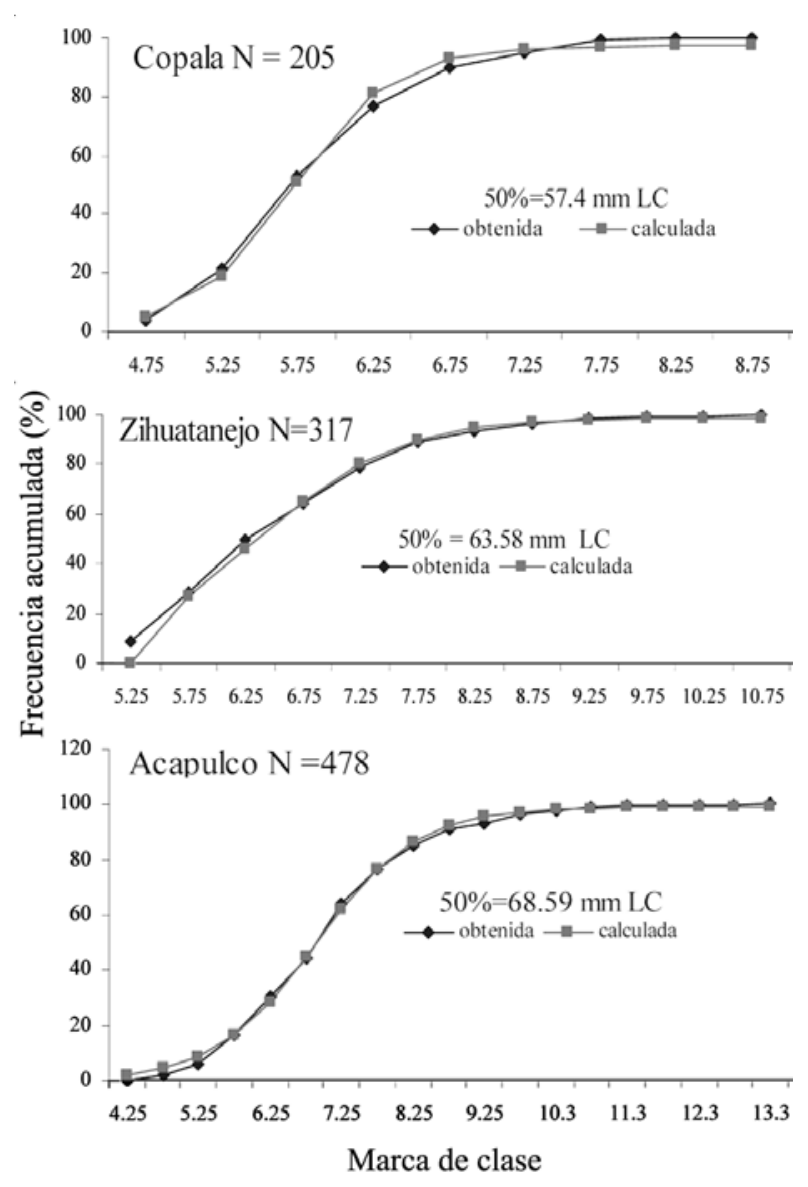

Figura 5

Talla mínimas de maduración sexual (mm LC) de las hembras de langosta espinosa $P$. inflatus en las tres zonas del litoral del estado de Guerrero, Pacífico mexicano

Minimum size of sexual maturity (mm LC) of the females of spiny lobster $P$. inflatus in the three areas in the Guerrero coast, Mexican Pacific

En el caso de las especies de climas tropicales como $P$. inflatus y $P$. gracilis, determinar la estación reproductiva y establecer periodos de veda se dificulta debido a que presentan actividad reproductiva con desoves múltiples durante todo el año (Weinborn 1977, Briones-Fourzán et al. 1981, Gracia 1985, BrionesFourzán \& Lozano-Álvarez 1992, Pérez-González et al. 1992) y aún cuando las hembras con huevos se presentan en diferentes porcentajes durante el ciclo anual, no llegan a presentar diferencias significativas entre estaciones (Briones-Fourzán \& Lozano-Álvarez 1992). Lo anterior ha conducido a considerar inválida la aplicación del período de veda o a sugerir la suspensión de esta temporada por lo menos de forma experimental en $P$. inflatus y P. gracilis (Briones-Fourzán et al. 1981, Gracia 1985). Sin embargo, la observación de diferencias estacionales en la fecundidad para $P$. inflatus en Zihuatanejo, con un aumento en otoño y una mayor talla de los huevos (Gracia 1985, Azpeita \& Cervantes 1995) sugieren una mayor actividad reproductiva en esta estación. Además, se considera que las condiciones apropiadas para la reproducción de esta especie se dan en otoño, debido a un alto contenido de materia orgánica en sedimentos así como un alto peso húmedo de los moluscos que le sirven de alimento (Lozano-Álvarez \& Aramori-Serrano 1997). Por otra parte, en otoño se registra una sobrepoblación de $P$. inflatus y $P$. gracilis, debido a una migración de individuos registrando densidades entre 4 y 8 veces mas altas que en invierno (Lozano-Álvarez et al. 1982).

Por lo antes señalado se ha considerado el otoño como la principal estación de desove para las hembras medianas y grandes de $P$. inflatus (entre 65 y $80 \mathrm{~mm}$ LC) con varios desoves durante la temporada reproductiva. Las langostas grandes inician los desoves en otoño mientras que las pequeñas crecen en otoño y desovan en invierno (Gracia 1985, Briones-Fourzán et al. 1981, Briones-Fourzán \& Lozano-Álvarez 1992). En $P$. argus también se ha registrado la producción máxima de huevos primero en langostas grandes (Bertelsen \& Matthews 2001).

En otras especies de langostas se ha observado que la relación entre fecundidad y longitud cefalotoráxica presenta diferencias en relación con la latitud como es el caso de Panulirus interruptus que presenta mayor fecundidad a menor latitud (Pineda et al. 1981) o diferencias en la fecundidad en diferentes áreas para una misma latitud como es el caso de P. guttatus (BrionesFourzán \& Contreras-Ortíz 1999) y P. argus (Fonseca-Larios \& Briones-Fourzán 1998, Bertelsen \& Matthews 2001).

Dado que no se cuenta con estudios de fecundidad en diferentes zonas de captura en el litoral de Guerrero, ni estudios recientes de fecundidad en Zihuatanejo, en este estudio se analiza la época de mayor actividad reproductiva a partir de los porcentajes de hembras y de la distribución de tallas en las diferentes estaciones del año.

Respecto a los porcentajes de hembras ovígeras, no se encontraron diferencias significativas en las diferentes estaciones del año, mientras que el análisis de su abundancia estacional por clases de talla sí mostró diferencias entre estaciones del año y entre las áreas de estudio. En Acapulco se destaca la presencia de un alto porcentaje de hembras mayores de $80 \mathrm{~mm}$ de LC con huevos en invierno y primavera, mientras que en Copala las hembras inferiores a $50 \mathrm{~mm}$ de LC presentan abundancias similares en otoño e invierno. 
En Acapulco, la presencia de ejemplares de tallas grandes en las capturas comerciales (160 mm de LC), fueron registradas en marzo y podría deberse a la presencia de algún evento que favoreció la migración de langostas grandes al área de pesca. Estas langostas estarían en desoves secundarios según las observaciones de Briones-Fourzán \& Lozano-Álvarez (1992). Sin embargo, la mayor frecuencia de langostas con huevos en las capturas totales mostró un máximo entre los 70 y $75 \mathrm{~mm}$ de LC, además se presentaron durante todo el año aunque con máximos en otoño y primavera.

Considerando la alta fecundidad y el mayor potencial de producción larval que tienen las hembras mayores a 80 mm de LC (Briones-Fourzán \& Lozano-Álvarez 1992), podría considerarse que la protección del recurso debería contemplar además de la veda en otoño otras medidas reguladoras. Varios investigadores han sugerido acciones para la protección de las hembras grandes como una talla máxima límite de captura o refugios especiales (Lozano-Álvarez et al. 1993), o el establecimiento de santuarios (Marx \& Herrnkind 1986). Sin embargo, se debe tomar en cuenta que en especies con desoves múltiples como P. inflatus (Briones-Fourzán \& LozanoÁlvarez 1992), P. japonicus y P. argus (Creaser 1950 e Ino 1950), conforme avanzan los desoves parciales, se presentan reducciones en la fecundidad que, en ocasiones, han sido considerables (70\%).

El rango de tallas en las capturas en Copala se encontró entre los 40 y $110 \mathrm{~mm}$ de LC con una mayor abundancia de langostas entre los 55 y $60 \mathrm{~mm}$ de LC. Las hembras de estas tallas y de 60 a $65 \mathrm{~mm}$ de LC presentan el mayor número de langostas ovígeras y ocurrieron durante todo el año y las tallas menores de 50 mm de LC presentan el máximo de hembras ovígeras en otoño e invierno. Por esta razón, dado que el invierno es considerado como la única temporada de reproducción de las hembras menores de $60 \mathrm{~mm}$ de LC, ya que empiezan a reproducirse después que las langostas grandes lo han hecho de acuerdo con Briones-Fourzán \& Lozano-Álvarez (1992), podría suponerse la existencia de fracciones de la población con tallas pequeñas pero de mayor edad reproductiva.

Mientras que el rango de capturas en Zihuatanejo (45130 mm de LC) fue igual al reportado por BrionesFourzán \& Lozano-Álvarez (1992) en las capturas comerciales de 1979-1980, la clase de mayor frecuencia de langostas, machos, hembras y hembras ovígeras mostró una disminución de 70-75 a 60-65 mm de LC. En esta pesquería, el número de hembras ovígeras en las capturas disminuyó (39,6 \%) respecto al estudio previo (51,5 \%), pero incrementó el porcentaje de estas hembras por clase de talla. La clase de talla de mayor frecuencia (60-65 mm de LC) presentó máximos en otoño.

Los resultados de este estudio apoyan que la veda para $P$. inflatus debiera mantenerse en otoño, época en la que se presenta el mayor número de hembras grávidas para la clase con mayor abundancia de organismos, por lo menos en Acapulco y Zihuatanejo; no obstante es evidente la falta de estudios sobre fecundidad en las diferentes zonas de esta pesquería y en diferentes tiempos, que permitan conocer si existen diferencias geográficas o temporales en este parámetro y su correlación con otras variables importantes, información que podría considerarse en el ordenamiento pesquero del recurso. Las diferencias observadas en la composición de tallas también llevan a formular la interrogante del origen del stock reproductor, áreas de crianza y migraciones de esta langosta, así como diferencias de crecimiento entre áreas de estudio.

\section{Talla de madurez sexual}

La talla de madurez sexual es considerada como la talla en la que el $50 \%$ de las hembras se encuentra en reproducción (Somerton 1980) y se determina para establecer la talla mínima legal de captura de las especies sometidas a explotación (Chubb 2000). En la pesquería de $P$. inflatus se aplicaba la talla mínima de captura de $85 \mathrm{~mm}$ de LC establecida para $P$. interruptus en las costas de Baja California en el Pacífico mexicano. Mas tarde fue modificada a $75 \mathrm{~mm}$ de LC por recomendaciones de Briones et al. (1981) a partir de estudios realizados en Zihuatanejo, Guerrero. A pesar de esta reducción es considerada superior a la talla mínima de madurez sexual (Chubb 2000).

Un estudio realizado en las costas de Sinaloa al norte del estado de Guerrero, reportan que la talla de primera madurez sexual fue de 69,53 (1990), 68,81 (1991) y 69,23 mm (1990-1991) y se recomienda como talla mínima de captura, 70 mm de LC (González 2002).

Las diferencias geográficas y la influencia de condiciones ambientales en la talla mínima de madurez sexual en langostas han sido documentadas en otras especies, como P. argus, en la región del Caribe, donde se han demostrado variaciones considerables (Lyons 1986). Al sur de África, Jasus lalandii presenta un lento crecimiento y disminuye la talla de madurez con respecto a las áreas del norte (Pollok 1986). Las reducciones en el crecimiento asociadas a las perturbaciones del Niño (1990-1993) condujeron a una reducción significativa de la talla de madurez (Pollok et al. 1977). También existen antecedentes del efecto de las condiciones ambientales en la composición de tallas y en la talla de madurez sexual (Mohan 1997). 
La disminución en la talla mínima de madurez sexual también ha sido reportada en poblaciones de langosta después de ser sometidas al efecto de la pesquería. DeMartini (1993) encontró para P. marginatus, en Hawai, una reducción de la talla mínima de madurez sexual y un incremento en la fecundidad en función de la intensidad de la explotación pesquera. Las pesquerías sobreexplotadas inducen a la reducción en la talla mínima de madurez sexual en las hembras de las poblaciones de langostas espinosas, probablemente como resultado de una rápida cosecha (Chubb 2000).

En el caso de la talla mínima de madurez sexual en las poblaciones de $P$. inflatus en las costas de Guerrero, es difícil establecer la causa de las diferencias observadas en este estudio, ya que no se tienen datos previos ni existen registros históricos de capturas que permitan estimar los cambios en la densidad de las poblaciones, así en Copala tenemos el registro mas bajo de la talla de madurez sexual de las tres áreas de esta pesquería, lo anterior puede ser atribuido a un lento crecimiento de las langostas en esa región o bien puede ser producto de una sobreexplotación del recurso. Algunos elementos a favor de un lento crecimiento pueden ser atribuidos a diferencias geográficas entre las zonas de captura y posibles diferencias ecológicas entre las regiones costeras de Guerrero y a que la rapidez de crecimiento de $P$. inflatus es altamente variable y es afectada por factores ambientales (Briones-Fourzán \& Lozano-Álvarez 1993).

En Acapulco, el valor (68,6 mm LC) es mas cercano al reportado para Sinaloa, al norte del área de estudio, y el de Zihuatanejo está por debajo de este valor por 5 $\mathrm{mm}$; existe la posibilidad de que esta reducción pudiera atribuirse al efecto de la pesca, aun cuando no se cuenta con registros de cambios en la densidad interanual del recurso, pero se cuenta con estudios previos que así lo sugieren como la reducción en la clase de talla de mayor aportación de organismos hembras y machos (Gracia 1985, Briones-Fourzán \& Lozano-Álvarez 1992, BrionesFourzán et al. 1981, Weinborn 1977).

\section{Implicaciones de manejo}

Por los resultados obtenidos en este estudio se considera necesario revisar la reglamentación sobre la talla mínima de captura para las distintas zonas en el litoral de Guerrero. Respecto a la variación geográfica de la talla mínima de madurez sexual se sabe que la talla límite no puede aplicarse igual en todas las áreas y marca la necesidad de emplear tallas mínimas de captura variables (Mohan 1977).

Se considera que la talla mínima debe establecerse por encima de la talla de madurez sexual para lograr una mayor protección de la población sin necesidad de reducir el esfuerzo de la captura. Para la pesquería de $P$. inflatus en Guerrero, debería mantenerse la talla mínima de captura de 75 mm de LC en Acapulco y Zihuatanejo, bajo una estricta vigilancia del cumplimiento de esta reglamentación y no incrementar el esfuerzo pesquero concediendo permisos de captura a nuevas cooperativas o incrementar el número de socios y artes empleadas. En Copala, el análisis parece indicar que es necesario reducir la talla mínima de captura a $65 \mathrm{~mm}$ de LC, disminuir el esfuerzo pesquero y emplear otras herramientas para determinar la talla límite apropiada.

\section{Agradecimientos}

Al Fideicomiso SAGARPA-CONACYT 2003 por proporcionar los fondos para el proyecto 'Estudio Biológico-Pesquero del recurso langosta y comparación de las artes empleadas para su captura en el litoral de Guerrero’ y al Instituto Politécnico Nacional (IPN) SAPI 20070625, a COFAA-IPN, por las becas a M. VillalejoFuerte. A los evaluadores y editores de este documento que ha mejorado sustancialmente gracias a sus comentarios, así como a los investigadores que contribuyeron con la información necesaria para desarrollar e interpretar este estudio.

\section{Literatura citada}

Azpeitia HA \& BJD Cervantes. 1995. Fecundidad de la langosta $P$. gracilis Street 1871, en las costas del sur de Sinaloa, México. Tesis de Licenciatura, Facultad de Ciencias del Mar, Universidad Autónoma de Sinaloa, Mazatlán, Sinaloa 62 pp.

Bertelsen RD \& TS Matthews. 2001. Fecundity dynamics of female spiny lobster (Panulirus argus) in a short Florida fishery and Dry Tortugas National Park lobster sanctuary. Marine and Freshwater Research 52: 1559-1565.

Briones-Fourzán P, E Lozano-Álvarez, A Martínez \& AS Cortés. 1981. Aspectos generales de la biología y pesca de las langostas en Zihuatanejo, Guerrero, México. Anales del Instituto de Ciencias del Mar y Limnología, Universidad Nacional Autónoma de México 8(1): 79-101.

Briones-Fourzán P \& E Lozano-Álvarez. 1992. Aspects of reproduction of Panulirus inflatus (Bouvier) and P. gracilis Streets (Decapoda: Palinuridae) from the Pacific coast of Mexico. Journal of Crustacean Biology 12: 41-50.

Briones-Fourzán P \& G Contreras-Ortiz. 1999. Reproduction of the spiny lobster Panulirus guttatus (Decapoda: Palinuridae) on the Caribbean coast of Mexico. Journal of Crustacean Biology 19: 171-179.

Briones-Fourzán P \& E Lozano-Álvarez. 2003. Factor affecting growth of the spiny lobsters Panulirus gracilis and P. inflatus (Decapoda: Palinuridae) in Guerrero, Mexico. Revista de Biología Tropical 51:165-174. 
Chubb CF. 2000. Reproductive biology: Issues for management. En: Phillips BF \& J Kittaka (ed), Spiny lobsters: Fisheries and Culture 14: 245-447. Blackwell Science, Oxford.

Creaser EP. 1950. Repetition of egg laying and number of eggs of the Bermuda spiny lobster. Proceedings of the Gulf and Caribbean Fisheries Institute 2: 30-31.

DeMartini EE, MD Ellis \& AV Honda. 1993. Comparisons of spiny lobster Panulirus marginatus fecundity, egg size, and spawning frequency before and later exploitation. Fishery Bulletin 91: 1-7.

Díaz-Arredondo MA \& SA Guzmán-del-Próo. 1995. Hábitos alimentarios de la langosta roja (Panulirus interruptus Randall, 1840) en Bahía Tortugas, Baja California Sur. Ciencias Marinas 21: 439-462.

Fonseca-Larios M \& P Briones-Fourzán. 1998. Fecundity of the spiny lobster Panulirus argus (Latreille, 1804) in the Caribbean coast of Mexico. Bulletin of Marine Science 63: 21-32.

González MBR. 2002. Madurez sexual durante la actividad reproductiva de la langosta Panulirus inflatus (Bouvier) en las costas de Sinaloa, México (1990-1991). Tesis de Licenciatura en Biología Pesquera, Universidad Autónoma de Sinaloa, Mazatlán, Sinaloa, 56 pp.

Gracia A \& BC Kensler. 1980. Las langostas de México: su biología y pesquería. Anales del Centro de Ciencias del Mar y Limnología de la Universidad Nacional Autónoma de México 7: 111-128.

Gracia GA. 1985. Variaciones estacionales en la fecundidad de la langosta Panulirus inflatus (Bouvier, 1895) (Crustacea: Decapoda: Palinuridae). Ciencias Marinas 11: 7-27.

Holthuis LB \& A Villalobos. 1962. Panulirus gracilis Streets y Panulirus inflatus (Bouvier) dos especies de langosta (Crustacea: Decapoda) de la costa del Pacífico de América. Anales del Instituto de Biología, Universidad Nacional Autónoma de México 32: 251-462.

Ino T. 1950. Observations on spawning cycle of Ise ebi (Panulirus japonicus (v. Siebold)). Bulletin of the Japanese Society of Scientific Fisheries 15: 725-7.

Lozano-Álvarez E, P Briones-Fourzán, L Santarelli \& A Gracia. 1982. Densidad poblacional de Panulirus gracilis Streets y $P$. inflatus (Bouvier) (Crustacea: Palinuridae) en dos áreas cercanas a Zihuatanejo, Guerrero, México. Ciencia Pesquera, 3: 61-73.

Lozano-Álvarez E, P Briones-Fourzán \& F Negrete-Soto. 1993. Occurrence and seasonal variations of spiny lobster,
Panulirus argus (Latreille), on the shelf outside Bahia Ascension, Mexico. Fishery Bulletin 91: 808-815.

Lozano-Álvarez E \& G Aramori-Serrano. 1997. Alimentación y estado nutricional de las langostas Panulirus inflatus y Panulirus gracilis (Decapoda: Palinuridae) en Guerrero, México. Revista de Biología Tropical 45: 453-461.

Lyons GW. 1986. Problems and perspectives regarding recruitment of spiny lobsters, Panulirus argus, to the South Florida fishery. Canadian Journal of Fisheries and Aquatic Science 43: 2099-2106.

Marx JM \& WF Herrnkind. 1986. Species Profiles: Life histories and environmental requirements of coastal fishes and invertebrates (South Florida). Biological Report 82: 11-61.

Mohan R. 1997. Size structure and reproductive variation of the spiny lobster Panulirus homarus over a relatively small geographic range along the Dhofar coast in the Sultanate of Oman. Marine and Freshwater Research 48: 1058-91.

Pérez-González R, L Flores-Campaña, AM Nuñez-Pasten \& A Ortega-Salas. 1992. Algunos aspectos de la reproducción en Panulirus inflatus (Bouvier) y P. gracilis Streets (Decapoda: Palinuridae) en el Sureste del Golfo de California, México. Investigaciones Marinas, Centro Interdisciplinario de Ciencias Marinas 7: 26-33.

Pineda BJ, CAJ Díaz de León \& OF Uribe. 1981. Fecundidad de la langosta roja Panulirus interruptus (Randall, 1842) en Baja California. Ciencia Pesquera 1: 99-118.

Pollok DE. 1986. Review of the fishery for and biology of the Cape rock lobster Jasus lalandii with notes on larval recruitment. Canadian Journal of Marine Science 43: 21072117.

Pollok DE, AC Cockcroft \& PC Goosen. 1977. A note on reduced rock lobster growth rates and related environmental anomalies en the southern Benguela, 1988-1995. South African Journal of Marine Science 18: 287-293.

Somerton DA. 1980. A computer technique for estimating the size of sexual maturity in crabs. Canadian Journal of Fisheries and Aquatic Science 47: 1488-1494.

Wayne WD. 2005. Bioestadística: bases para el análisis de las ciencias de la salud. 915 pp. Editorial Limusa, México.

Weinborn JA. 1977. Estudio preliminar de la biología, ecología y semicultivo de Palinúridos de Zihuatanejo, Gro., México. Panulirus gracilis Streets y Panulirus inflatus (Bouvier). Anales del Instituto de Biología, Universidad Nacional Autónoma de México 4: 1-248. 